\section{BACTERIAL CONTAMINATION OF INTENSIVE CARE UNITS (NICU) AND DETERMINATION OF ANTIBIOTICS RESISTANCE PATTERNS IN ISOLATES IN HAMADAN, WEST OF IRAN}

\author{
R. Yousefi Mashouf, M. Momeni, N. Pezeshki
}

Hamadan University of Medical Sciences, Hamadan, Iran

Background and aim: Bacterial contamination in hospitals is one of the major problems in hospitals that cause serious damage to society. The aims of this study was the evaluation of bacterial contamination of intensive care units (NICU) and determination of antibiotics resistance patterns in isolated bacteria in 2008 .

Material and methods: This was a cross-sectional study that 400 samples were randomly collected from environments and apparatus of intensive care units including washing sink, floor of wards, beds of patients, oxygen mask, incubator, infant scale, suction and staff fingers. The samples were inoculated in EMB and Blood agar by wet swabs and transferred to laboratory for identification. Strains were selected and cultured on Mulerhinton agar for antibiogram tests by NCCLS method. Data was gathered through a questionnaire and analyzed by SPSS 13 software.

Results: The average rate of bacterial contamination of NICU of Fatemihe hospital was $79.5 \%$. The most contaminated places were washing sink (100\%), suction $(74 \%)$ and the lowest was phototherapy (35\%) and oxygen mask (44\%), respectively. The most bacteria isolated were as follow: Staphylococcus epidemidis (17\%), Bacillus subtilis (12.5\%), Acinetobacter baumannii (11.3\%) and E. coli (8.2\%). Most of isolates (60\%-90\%) were sensitive against ceftriaxone, vancomycin, gentamicin and ciprofloxacin, whereas most of them were resistant to ampicillin, erythromycin and cephalexine.

Conclusion: Our results showed the considerable bacterial contamination (79.5\%) of NICU in particular with Acinetobacter baumannii and the high drug resistance in strains isolated from hospital, it seems that sterilization and disinfection methods in hospitals were not performed correctly.

\section{4}

\section{H1N1 IN CHILDREN REQUIRING INTENSIVE TREATMENT; THE ROTTERDAM EXPERIENCE}

\author{
R. Kuppens, P. Fraaij, R.-J. Houmes, C. Buysse
}

Department of Paediatrics, Division of Paediatric Intensive Care, Erasmus MC - Sophia Children's Hospital, Rotterdam, The Netherlands

Objective: To describe the characteristics of children with $\mathrm{H} 1 \mathrm{~N} 1$ influenza who required PICU (paediatric intensive care unit) treatment.

Patients and methods: We reviewed the charts of all children with laboratory-confirmed H1N1 influenza requiring PICU admission between September 2009 and March 2010 in the Erasmus MC-Sophia Children's Hospital, the Netherlands.

Results: 12 children were admitted to our PICU, whereas 1 died before transport to our hospital due to cardiorespiratory failure. Of these 12 children (50\% boys, mean age 10.8 years), 7 (58\%) had clinical significant previous medical conditions. All 12 presented with fever and flu-like symptoms. Mean interval between onset of symptoms and PICU admission was 2 days (0-5 days). PICU treatment consisted of oseltamivir $(n=12,100 \%)$, high flow oxygen through a non-rebreathing mask ( $n=5,42 \%$, mean duration 1 day), invasive mechanical ventilation ( $n=1,8 \%$, duration 2 days) and extracorporeal membrane oxygenation (ECMO) because of severe cardiorespiratory failure $(n=6$, $50 \%$ ). The latter 6 children were referred by other Dutch PICU tertiary centres for ECMO therapy. Of these 6 children, $4(66 \%)$ had clinical significant previous medical conditions. Four of the 6 were discharged alive from the PICU after a mean ECMOduration of 7.5 days, whereas 2 died after a mean ECMO-duration of 31 days because of refractory respiratory failure with a staphylococcus-infection.

Conclusions: The majority of children with H1N1 admitted to our PICU were teenagers and had clinical significant previous medical conditions. 50\% were referred by other PICU for ECMO treatment. Overall mortality was high (23\%). 